\title{
A grand challenge for applied genetic epidemiology: putting the human genome in context
}

\section{Anthony Gean Comuzzie* \\ Department of Genetics, Texas Biomedical Research Institute, San Antonio, TX, USA \\ ${ }^{*}$ Correspondence: tony@sfbrgenetics.org}

The last two decades witnessed rapid and profound expansion in the field of human genetics, driven in large part by the completion of the Human Genome Project. While the Human Genome Project achieved its goal of mapping the physical location of the approximately 20,000 to 25,000 genes that make up our genome, it has left open the most challenging biological question "How do these genes impact who we are?" As a result, the role of genetic epidemiology is to take the information gained from the Human Genome Project and combine it with carefully characterized phenotypic data from well defined populations to identify not only which genes impact everything from physical appearance, to behavior, to disease susceptibility, but how they interact with the environment.

Not only did the completion of the Human Genome project provide us with information regarding the basic architecture of our genome, it also gave rise to impressive advances in molecular technologies which now make it possible to employ high resolution methods for the assessment of genetic variation at a population level in a high through-put and cost-effective manner. By example it is now routine to assess over a million single nucleotide polymorphisms (SNPs) on thousands of individuals within a single study, and then combining them into meta-analyses with hundreds of thousands of individuals. Beyond simply examining nucleotide variation, it is now feasible to assess other aspects of genomic variation including whole transcriptome profiling, genome-wide epigenetic modifications, and a burgeoning capacity for whole genome sequencing on an individual basis. Consequently, a major challenge now facing the field of genetic epidemiology is how to apply this rapidly expanding plethora of genetic data in meaningful ways that will further our understanding of human biology.

Currently much of the effort in genetic epidemiology is still largely focused on attempting to identify which genes influence which phenotypes, largely through genome-wide efforts employing either case/control study designs utilizing association methods with a few investigators still using linkage approaches. While the identification of the key genes involved in the expression of a phenotype, particularly for those involved in mediating disease risk, is an important endeavor, it only represents a first step. It is highly doubtful that any gene will exert its effects completely in isolation, but rather will have its action modulated by a wide range of other genetic and also environmental factors. The identification of such environmental factors and the deciphering of how they impact the action of genes is a fundamental objective of genetic epidemiological analyses. Therefore, as the diversity, as well as shear amount, of genetic information continues to accumulate, the thoughtful definition, and quantification of key environmental factors must also keep pace if we are to truly understand how the critical interaction between genes and environment give rise to the phenotypic variation we observe at the population level.

The field of genetic epidemiology holds a critical position within human genetics as it provides a key component of the epistemological framework by which we attempt to put the human genome into a meaningful biological context. Therefore, the grand challenge for the field of genetic epidemiology will be to move beyond simple gene identification to include how identified genes interact with the environment in which these genes find themselves, as well as how to make creative and insightful use of the vast wealth of genomic data which is now being produced, and all done in an effort to understand the connection between the genes and their physical manifestation in the individual. It will be the goal of Frontiers in Applied Genetic Epidemiology to provide a forum for the presentation of creative research seeking to further our understanding of not only how genomic variation, assessed by multiple methods, impact phenotypic expression, but how genes interact with the environment to give rise to the patterns of variation and complexity we observe at the population level. We are eager to meet this challenge in our own work and welcome all those who wish to join us in this important scientific endeavor.

Received: 17 February 2011; accepted: 17 February 2011; published online: 03 March 2011.

Citation: Comuzzie AG (2011) A grand challenge for applied genetic epidemiology: putting the human genome in context. Front. Gene. 2:10. doi: 10.3389/fgene.2011.00010 This article was submitted to Frontiers in Applied Genetic Epidemiology, a specialty of Frontiers in Genetics. Copyright $\odot 2011$ Comuzzie. This is an open-access article subject to an exclusive license agreement between the authors and Frontiers Media SA, which permits unrestricted use, distribution, and reproduction in any medium, provided the original authors and source are credited. 